\title{
Herpes simplex virus type-specific serology: Where does it fit in the diagnostic armamentarium?
}

\author{
Todd F Hatchette MD FRCPC
}

W ith data supporting the use of suppressive antiviral therapy to reduce transmission of herpes simplex virus (HSV) between discordant couples (1), and with the availability of more sensitive and specific serological assays, there has been increasing emphasis on accurate diagnosis of HSV infection in an effort to control the genital herpes epidemic. In this issue of The Canadian Journal of Infectious Diseases 83 Medical Microbiology, Ratnam et al (2) review herpes diagnostics and make suggestions for their use by Canadian clinicians. Clearly, virus isolation or detection of HSV type 1 (HSV-1) or HSV type 2 (HSV-2) viral DNA by molecular methods, such as polymerase chain reaction (PCR) from a genital lesion, is diagnostic of genital herpes. The speed and enhanced sensitivity of PCR compared with culture make it the test of choice for the diagnosis of active disease (3). Although PCR can detect asymptomatic shedding, viral detection tests are generally only of value when genital lesions are present. In the absence of lesions, some experts have suggested the use of type-specific serology (TSS) to identify individuals who are infected with HSV.

The rationale for serological testing is to identify asymptomatic HSV infection. Until recently, HSV serology was unhelpful because it could not accurately differentiate antibodies to HSV-2 (almost exclusively as a result of genital herpes) from HSV-1 (predominantly generated in response to an orolabial infection). HSV TSS uses commercially available tests that have the ability to accurately differentiate or 'type' antibody responses generated by HSV-1 from HSV-2 infection. However, interpretation of the results requires an understanding of the performance characteristics of the test, as well as the prevalence of the infection in the population in which testing is being applied.

What does a positive HSV TSS mean? There are data to suggest that knowledge of an individual's HSV-2 status can allow for disclosure and implementation of suppressive antiviral therapy to reduce the risk of transmission to partners $(1,4)$. Certainly, a positive HSV-2 result generally equates to a diagnosis of genital herpes. However, because specificity of TSS is not $100 \%$, false-positive results will occur. For example, when used in pregnant women, the HerpeSelect-2 kit (Focus Technologies, USA) has a specificity of $96 \%$, which means that of every 100 positive tests, four of these will be falsely positive (Table 1, Ratnam et al). If the overall prevalence of HSV-2 antibodies in pregnant women in Newfoundland and Labrador is $6 \%$, the positive predictive value (PPV) of this test is only 61\%. Can a PPV of $61 \%$ allow a physician to confidently say that the patient is infected with HSV-2 and no longer at risk for primary infection?

Conversely, a negative HSV-2 result does not rule out the diagnosis of genital herpes because the patient may have an HSV-1 infection to account for symptoms. In fact, as acknowledged by Ratnam et al, there is an increasing prevalence of HSV-1 as the cause of genital herpes in some regions of the country. In Nova Scotia, the majority of genital swabs in young people are positive for HSV-1, not HSV-2. However, what does a positive HSV-1 serology result mean? This may represent an orolabial infection, and not genital herpes at all. The absence of a history of cold sores is unhelpful because as with genital infections, many people who have orolabial infections are unaware that they have been infected. Differentiation of these two possibilities is often impossible, making a positive HSV-1 result unhelpful. Ratnam et al suggest that a positive result provides the opportunity for counselling regarding transmission via oral and genital sex. However, if the patient has HSV-1 antibodies generated by orolabial HSV-1 infection, to suggest that these antibodies protect them from subsequent HSV-1 genital infection may not be completely accurate. Instances of autoinoculation of HSV-1 from an 
orolabial lesion to the genitals suggest that the antibodies are not completely protective. Thus, how does this knowledge of HSV serostatus change the patient's counselling or management?

While the recommendations on the use of serological tests focus on selective populations thought to benefit the most from serodiagnosis, how exactly these tests should be used remains controversial (5-12). Although Ratnam et al have provided a comprehensive review of the literature on HSV TSS, a number of recommendations merit further consideration.

- Ratnam et al suggest that "TSS should be performed or repeated on specimens collected at least six weeks after exposure to ensure that the antibody response has occurred". However, it is unclear what is to be considered an exposure. Because the prevalence of HSV-2 in many areas may be $20 \%$ and for HSV-1 it could be $50 \%$ to $80 \%$ (13), there is a one in five chance that unprotected intercourse, or a greater than one in two chance that oral sex, with any new partner would equate to HSV exposure. Given that infected individuals only shed the virus intermittently, the risk would obviously not be this high. However, it is unclear what the risk of acquiring HSV is from one sexual encounter or how many people would need to be screened to diagnose one infection.

- Ratnam et al also suggest that HSV-2 TSS be offered to those diagnosed with HIV, a measure that "could provide the opportunity to improve health outcome and also serve as an adjunct to risk-reduction counselling". The prevalence of HSV-2 in HIVinfected individuals is indeed higher than the general population and, thus, the PPV of the test will be higher (13). Data also suggest that HSV-2 facilitates the acquisition of HIV and that suppression of HSV can reduce the HIV viral load, potentially reducing the risk of transmission of HIV (14-16). Chronic suppressive therapy using daily valacyclovir has been shown to decrease plasma and genital levels of HIV RNA in women dually infected with HSV-2 and HIV (16). However, whether knowledge of patients' HSV serostatus translates into adopting risk-reduction strategies is unclear and unproven. For example, if an HIV-infected patient has not modified behaviours on the basis of his or her HIV status, is it realistic to expect that the documentation of HSV-2 infection would yield behavioural changes?

- In regard to offering HSV TSS testing as an opportunity to improve the health of HIV-infected individuals, I would speculate that the majority of those in whom HSV-2 infection has had a significant negative impact have identifiable outbreaks that can be diagnosed with molecular methods. There is no indication for treatment of asymptomatic HSV in persons with HIV. In fact, HSV-suppressive therapy with acyclovir in immunosuppressed individuals is associated with higher rates of resistance than in those who are immunocompetent (17). Thus, it warrants consideration that suppressive therapy in an immunosuppressed, asymptomatic individual may not be reasonable and in the long-term, could be harmful.

Ratnam et al have put together a comprehensive and timely review. However, multiple issues need consideration when choosing a test and interpreting its result. With any diagnostic test, the prevalence of the disease in the population is essential to interpreting the test result. Clinicians need to be aware of the test limitations and need to consider whether the results influence the treatment or outcome. If the results do not influence the outcomes or management, then testing is a waste of finite health resources and is not indicated. We must also ensure that the reason for ordering the test is evidence-based and not driven by marketing campaigns.

Fundamentally, I see guidelines as an important way to standardize and ensure appropriate testing. Although I understand the basis for the recommendations made by Ratnam et al, I still struggle with identifying where HSV TSS fits into the diagnostic armamentarium of HSV infection; discussions with other clinicians suggest that I am not alone. In the clinical laboratory, we do not reliably get clinical information to help guide decisions on whether the test requested is appropriate. I am unsure how useful HSV TSS would be in the population my laboratory serves, in which the prevalence of genital infection due to HSV-1 is higher than that of HSV-2. A cost-effectiveness analysis taking into consideration the increased costs of testing, the cost of the subsequent interventions (suppressive therapy) and whether the testing influences outcomes needs to be considered if these suggestions are translated into public health policy. My apprehension is that HSV TSS will start in select circumstances but slip into broader practice without the appropriate evidence or cost-benefit analysis to support its use until it becomes a part of routine screening of asymptomatic patients presenting for a sexual health check up or even as a regular part of the annual medical examination.

ACKNOWLEDGEMENTS: The author thanks Drs Lynn Johnston and Jill Hatchette for reviewing the present manuscript and for their helpful comments. Dr Hatchette has no conflicts of interest to declare.

\section{REFERENCES}

1. Corey L, Wald A, Patel R, et al; Valacyclovir HSV Transmission Study Group. Once-daily valacyclovir to reduce the risk of transmission of genital herpes. N Engl J Med 2004;350:11-20.

2. Ratnam S, Severini A, Zahariadis G, Petric M, Romanowski B. The diagnosis of genital herpes - beyond culture: An evidencebased guide for the utilization of polymerase chain reaction and herpes simplex virus type-specific serology. Can J Infect Dis Med Microbiol 2007;18:233-40.

3. Espy MJ, Uhl JR, Sloan LM, et al. Real-time PCR in clinical microbiology: Applications for routine laboratory testing. Clin Microbiol Rev 2006;19:165-256. (Erratum in 2006;19:595).

4. Urato AC, Caughey AB. Universal herpes screening in pregnancy: Not recommended and potentially harmful to patients. Am J Obstet Gynecol 2007;196:e15.

5. Wald A, Krantz E, Selke S, Lairson E, Morrow RA, Zeh J. Knowledge of partners' genital herpes protects against herpes simplex type 2 acquisition. J Infect Dis 2006;194:42-52.

6. Guerry SL, Bauer HM, Klausner JD, et al. Recommendations for the selective use of herpes simplex virus type 2 serological tests. Clin Infect Dis 2005;40:38-45. 
7. Arvin AM. Debate: The argument against. Should all pregnant women be offered type-specific serological screening for HSV infection? Herpes 2002;9:48-50.

8. Malkin JE. Herpes simplex virus: Who should be tested? Herpes 2002;9:31.

9. Mindel A, Taylor J. Debate: The argument against. Should every STD clinic patient be considered for type-specific serological screening for HSV? Herpes 2002;9:35-7.

10. Patrick DM, Money D. Debate: The argument for. Should every STD clinic patient be considered for type-specific serological screening for HSV? Herpes 2002;9:32-4.

11. Kinghorn GR. Debate: The argument for. Should all pregnant women be offered type-specific serological screening for HSV infection? Herpes 2002;9:46-7.

12. Baker DA. Risk factors for herpes simplex virus transmission to pregnant women: A couples study. Am J Obstet Gynecol 2005;193:1887-8
13. Smith JS, Robinson NJ. Age-specific prevalence of infection with herpes simplex types 2 and 1: A global review. J Infect Dis 2002;186:S3-28.

14. Corey L, Wald A, Celum CL, Quinn TC. The effects of herpes simplex virus- 2 on HIV-1 acquisition and transmission: A review of two overlapping epidemics. J Acquir Immune Defic Syndr 2004;35:435-45

15. Lingappa JR, Celum C. Clinical and therapeutic issues for herpes simplex virus-2 and HIV co-infection. Drugs 2007;67:155-74.

16. Nagot N, Ouédraogo A, Foulongne V, et al; ANRS 1285 Study Group. Reduction of HIV-1 RNA levels with therapy to suppress herpes simplex virus. $\mathrm{N}$ Engl J Med 2007;356:790-9.

17. Bacon TH, Levin MJ, Leary JJ, Sarisky RT, Sutton D. Herpes simplex virus resistance to acyclovir and penciclovir after two decades of therapy. Clin Microbiol Rev $2003 ; 16: 114-28$ 


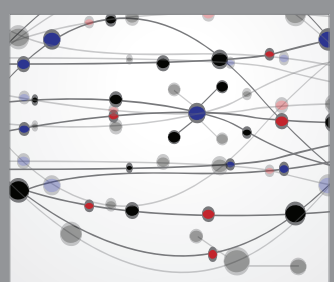

The Scientific World Journal


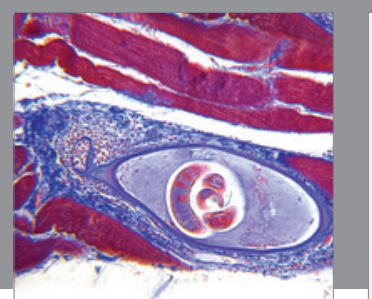

Gastroenterology Research and Practice

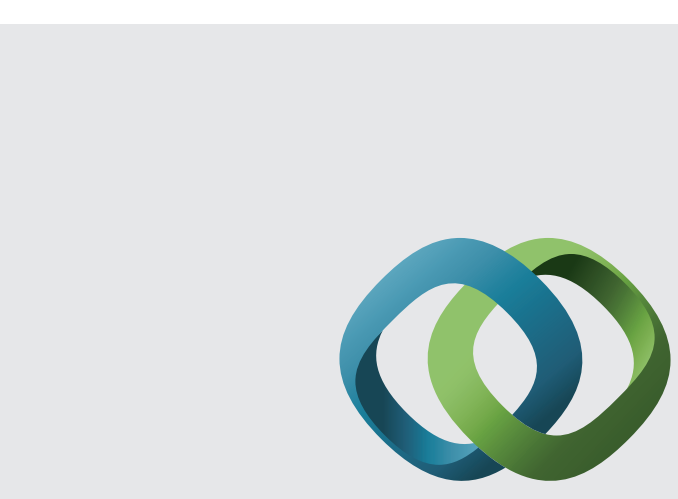

\section{Hindawi}

Submit your manuscripts at

http://www.hindawi.com
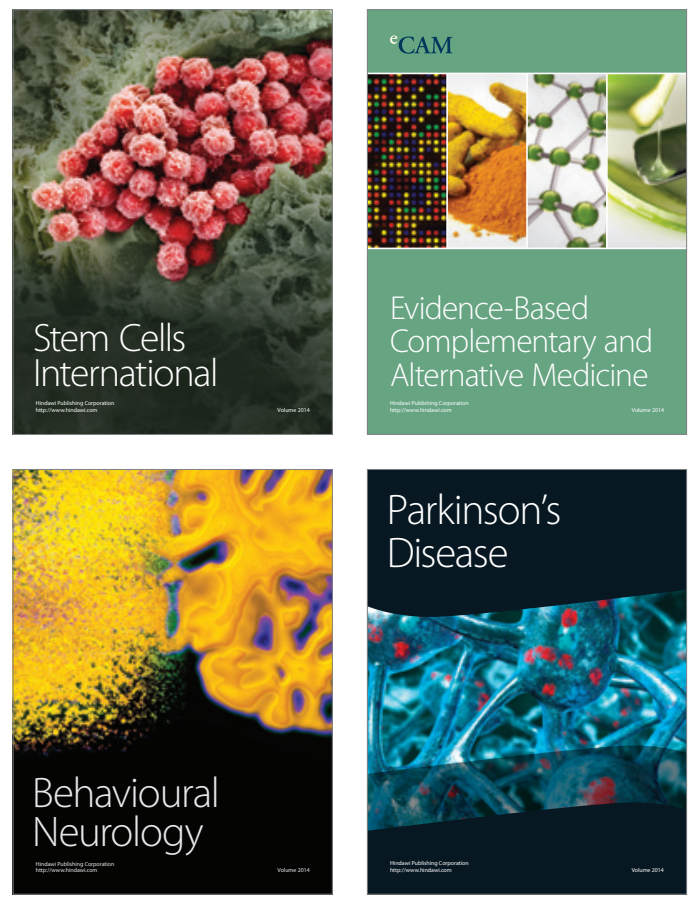
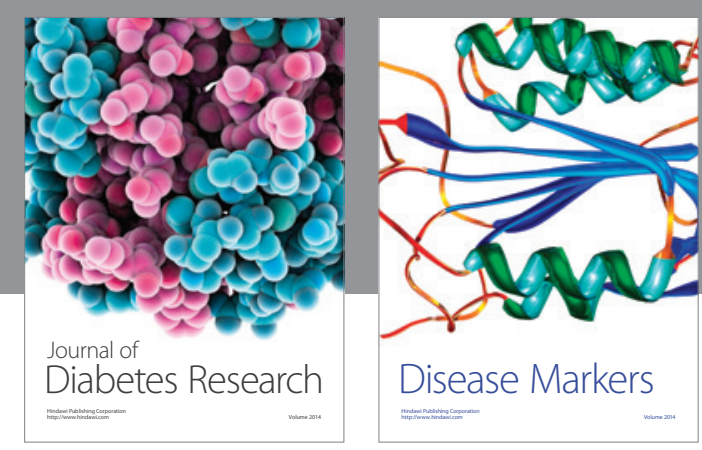

Disease Markers
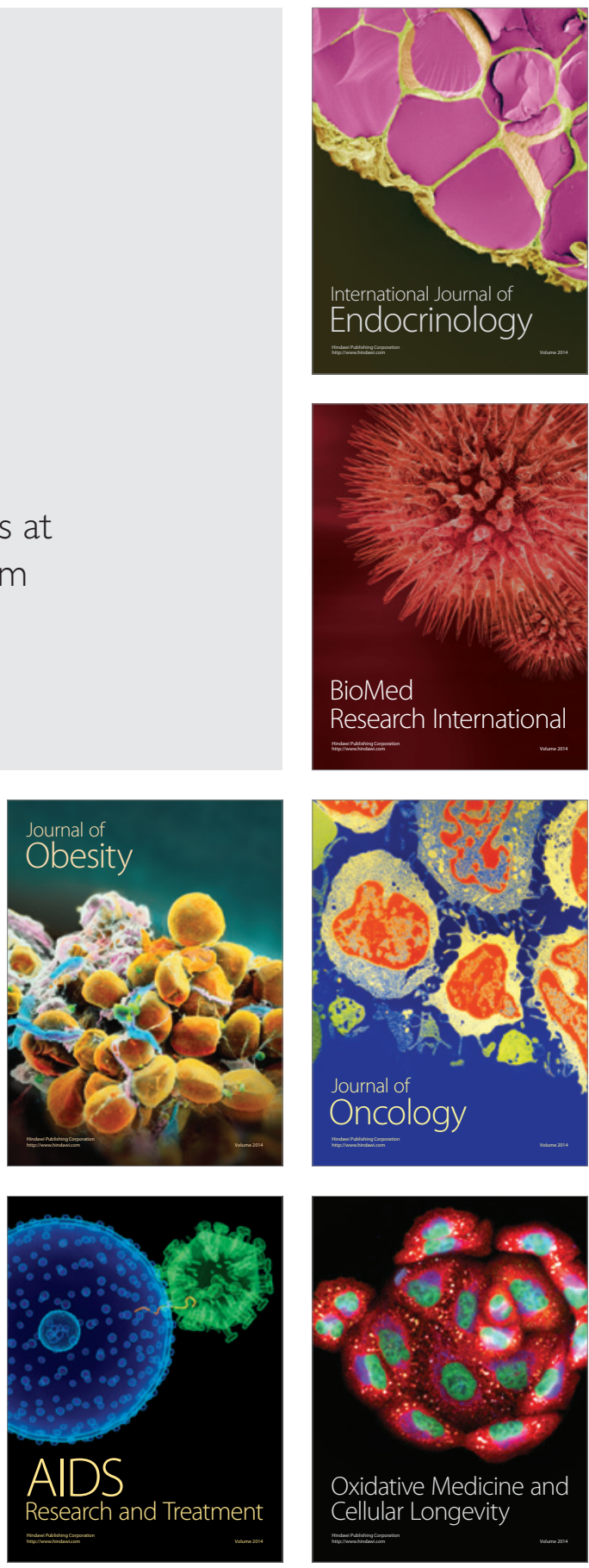